\title{
MicroRNA-195 Suppresses the Progression of Pancreatic Cancer by Targeting DCLK1
}

\author{
Bin Zhou Chuandong Sun ${ }^{\mathrm{a}}$ Xiao Hu Hanxiang Zhan ${ }^{\mathrm{b}}$ Hao Zou ${ }^{\mathrm{a}}$ Yujie Feng ${ }^{\mathrm{a}}$ \\ Fabo Qiu ${ }^{a}$ Shun Zhang ${ }^{a}$ Liqun Wu ${ }^{a}$ Bingyuan Zhang ${ }^{a}$
}

aDepartment of Hepatopancreatobiliary Surgery, the Affiliated Hospital of Qingdao University, Qingdao, Shandong Province; bepartment of General Surgery, Qilu Hospital, Shandong University, Jinan, Shandong Province, China

\section{Key Words}

Pancreatic cancer $\cdot$ DCLK1 $・$ MiR-195 $•$ Proliferation $•$ Metastasis

\begin{abstract}
Background/Aims: Doublecortin-like kinase 1 (DCLK1) is emerging as a tumor-specific stem cell marker in pancreatic cancer (PC). MicroRNA-195 (miR-195) plays an important role in many types of tumors. However, the roles of DCLK1 in cancer and miRNAs that directly regulate $D C L K 1$ have not been elucidated. The goal of this study is to assess the effects of $m i R-195$ on inhibiting DCLK1 and to clarify the regulating mechanism of miR-195-DCLK1 in PC cells. Methods: The expression of DCLK1 protein and miR-195 in PC tissues and adjacent healthy pancreatic tissues was detected by Western blot and quantitative reverse transcription polymerase chain reaction (qRT-PCR), respectively and the correlation between overall survival of PC patients and expression of DCLK1 was measured by Kaplan-Meier analysis. Bioinformatics tools were used to identify the target gene of miR-195. Effects of miR-195 and DCLK1 on proliferation and cell cycle of PC cells were analyzed by MTT, colony formation assays and flow cytometry. Transwell and wound-healing experiments were employed to examine the cellular migration and invasion. A xenograft mouse model was also used to test the effects of miR-195 on tumor growth and metastasis in vivo. Results: The expression level of DCLK1 and miR-195 shows an inverse correlation in PC tissues and cell lines. A higher DCLK1 level is associated with higher TNM (tumor, node, and metastasis) stage, higher rate of lymph node metastasis, and poor survival. Luciferase reporter assay shows that miR-195 directly targets $D C L K 1$. Overexpression of miR-195 inhibits proliferation, migration and invasion of PC cells, whereas downregulation of $m i R-195$ has an opposite role. These actions were similar to the effects of knockdown and overexpression of $D C L K 1$, respectively. Conclusions: These data suggest that miR-195 has tumor suppressor roles in PC by targeting DCLK1. MiR-195-DCLK1 pathway may provide insight into PC progression and represent a novel, promising diagnostic and therapeutic target for PC.
\end{abstract}

B. Zhou and C. Sun contributed equally to this work.

Bin Zhou

and Bingyuan Zhang
Department of Hepatopancreatobiliary Surgery, the Affiliated Hospital of Qingdao

University, Qingdao, Shandong Province (China)

E-Mail binalin@163.com, bingyuanzhang@126.com

\section{KARGER}




\section{Cellular Physiology Cell Physiol Biochem 2017;44:1867-1881

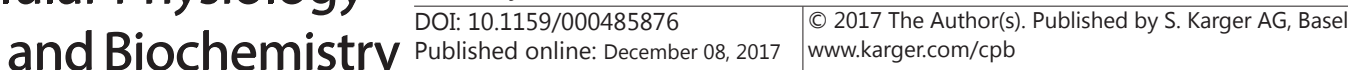 \\ Zhou et al.: MiR-195 Targets DCLK1 in PC}

\section{Introduction}

Pancreatic cancer (PC) is a disease associated with high mortality, with a 5-year overall survival rate of less than $5 \%$ [1] because of its late presentation, early metastasis, and unresponsiveness to most treatment options [2]. Only $15 \%$ to $20 \%$ of PC tumors are resectable at the time of diagnosis. In a large proportion of patients with PC, locally advanced or metastatic disease is diagnosed at the time of presentation because PC usually causes no symptoms in its early stage $[3,4]$. Nevertheless, PC responds poorly to both chemotherapy and radiation therapy [5]. Therefore, a better understanding of the molecular mechanisms underlying PC development will be useful to identify novel detection biomarkers and to improve current therapeutic strategies.

In our preliminary studies, through exome capture sequencing and bioinformatics analysis, we found that doublecortin-like kinase 1 (DCLK1) is a specific molecular candidate associated with PC metastasis [6]. DCLK1 is a microtubule-binding member of the calmodulindependent kinase family and is overexpressed in pancreatic intraepithelial lesions as well as surgical resection specimens of PC [7-9]. Previous studies have demonstrated the therapeutic effects of DCLK1 in cancer. Inhibiting DCLK1 with small interfering RNA (siRNA) suppresses epithelial-to-mesenchymal transition of PC cells through regulating important microRNAs (miRNAs) [8]. However, the functional role of miRNAs on targeting DCLK1 in PC has not been clarified.

A recent study has shown that deregulated expression of series miRNAs correlates with PC development [10]. Through the online tools and luciferase assay, we found that microRNA-195 (miR-195) directly targets DCLK1. MiR-195 is an important member of the miR-15a, $-15 b,-16,-195$, and -497 families, which play an important role in the occurrence and developmental progress of many tumors and in regulation of malignant biological behaviors [11-14]. Nevertheless, the role of miR-195 in PC development and especially the potential targets of this miRNA in PC cells remain unknown.

In this study, we found that DCLK1 exhibited a higher level, whereas miR-195 was significantly downregulated in human PC tissues and cell lines, in which DCLK1 and miR-195 have an inverse relationship. Both high level of DCLK1 and low level of miR-195 in PC tissues are associated with poor survival of patients with PC. We also clarified the effects of miR195 on proliferation and metastasis of PC cells by using in vitro assays and animal models. Furthermore, we confirmed that miR-195 exerts its tumor suppressor effects by inhibiting DCLK1 in PC cells. Our findings highlight that miR-195 functions as a tumor suppressor in PC and implicate miR-195-DCLK1 as a potential diagnostic and therapeutic target for PC.

\section{Materials and Methods}

\section{Patient tissue samples and PC cell lines}

The human PC tissues and adjacent healthy pancreatic tissues were obtained from the Affiliated Hospital of Qingdao University and Qilu Hospital between January 2011 and May 2016 with patients' informed consent. The category of PC samples was confirmed by pathological analysis. The use of these samples was approved by the Clinical Research Ethics Committee of Qingdao University (Qingdao, People's Republic of China [PRC]). All samples were immediately snap-frozen in liquid nitrogen and stored at $-80^{\circ} \mathrm{C}$ until used. None of the patients received chemotherapy or radiotherapy before the surgical excision. Five PC cell lines (BxPC-3, PANC-1, SW1990, MIAPaCa-2, and AsPC-1) and human normal pancreatic duct epithelial cells (HPDE6-C7) were purchased from the Tianjin Sai'er Biotechnology Co. Ltd (Tianjin, PRC).

\section{Immunohistochemistry staining}

Immunohistochemistry assay was performed by BenchMark XT automated slide-staining instrument (Ventana, Arizona, USA) with Ultraview Universal DAB Detection Kit (Ventana, Arizona, USA) after deparaffinization, rehydration, and antigen retrieval. After primary antibody incubation, the sections were incubated with anti-mouse horseradish peroxidase labeled polymer (Roche, Arizona, USA) for $30 \mathrm{~min}$ 


\section{Cellular Physiology Cell Physiol Biochem 2017;44:1867-1881

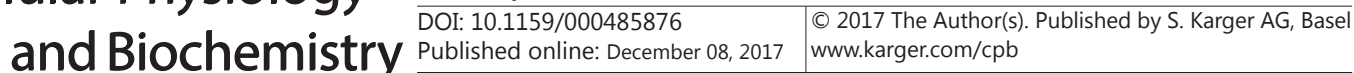 \\ Zhou et al.: MiR-195 Targets DCLK1 in PC}

at room temperature, and then developed with diaminobenzidine. All slides were counterstained with hematoxylin. For DCLK1 staining, the reactivity assessed was cytoplasmic. All micrographs were taken with a Nikon microscope equipped with a digital color camera and software. The immunostaining was evaluated by a pathologist who was blinded to patient prognosis and clinicopathological outcomes.

\section{Cell culture and transfection}

All cells were grown in RPMI 1640 (GIBCO BRL, USA) supplemented with 10\% fetal bovine serum (FBS), $100 \mathrm{IU} / \mathrm{mL}$ of penicillin, and $100 \mu \mathrm{g} / \mathrm{mL}$ of streptomycin, and incubated at $37^{\circ} \mathrm{C}$ in a humidified chamber supplemented with $5 \% \mathrm{CO}_{2}$. Transfection was performed with Lipofectamine 2000 Reagent (Invitrogen) following the manufacturer's protocol. Spiked red fluorescent protein (RFP)-expressing vector was used to monitor transfection efficiency.

\section{The predication of miRNA targeting gene}

The miR-195 targets were predicted on the calculations of mirSVR scores by the computer-aided algorithms of PicTar and TargetScan Release 5.1.

\section{Luciferase-reporter assay}

PANC-1 cells were cotransfected using $0.2 \mu \mathrm{g}$ of luciferase reporter vector with a wild-type or mutant DCLK1 3'UTR) in 48-well plates and miR-195 mimics or mimics control. The assay was normalized with 0.05 $\mu \mathrm{g}$ of the RFP expression vector pDsRed2-N1 (Clontech, USA). The cells were lysed with RIPA lysis buffer (0.15 M NaCl, 0.05 M Tris/HCl pH 7.2, 1\% Triton X-100, 0.1\% SDS) 48 hours later. The luciferase activity and RFP intensity were detected with an F-4500 Fluorescence Spectrophotometer (Hitachi, Japan).

\section{RNA isolation and quantitative reverse transcription polymerase chain reaction ( $q R T-P C R$ )}

Total RNA from cultured cells or surgical tissues was extracted using Trizol reagent (Invitrogen, Carlsbad, CA, USA) according to the manufacturer's instructions. qRT-PCR was performed using the Allin-One ${ }^{\mathrm{TM}}$ miRNA qRT-PCR detection kit (GeneCopoeia, Rockville, MD, USA) for miR-195 and small nuclear RNA U6 as an endogenous control. The relative expression level of mRNA was detected by SYBR Green qRT-PCR assay (Bio-Rad Laboratories Inc, Hercules, CA, USA), and $\beta$-actin was used as an endogenous control. All qRT-PCR procedure was performed on an ABI 7500 thermocycler (Thermo Fisher Scientific, Waltham, MA, USA). Primers used were as follows: $\beta$-actin, 5'-AGTGTGACGTGGACATCCGCAAAG-3' (forward), 5'-ATCCACATCTGCTGGAAGGTGGAC-3' (reverse); DCLK1， 5'-CCAGCTCAGTTAATGGAAC-3' (forward), 5'-GGACGCAAGTGACGTAGA-3' (reverse). The specific primers for miR-195 (HmiRQP0376) and U6 (HmiRQP9001) were purchased from RiboBio (Guangzhou, PRC). The relative expression levels were calculated using the $2^{-\Delta \Delta c t}$ method.

\section{Western blot analysis}

Cells were lysed using the protein-extraction reagent radioimmunoprecipitation assay (GIBCO) supplemented with protease inhibitors. Then, approximately $50 \mu \mathrm{g}$ protein extractions were subject to $10 \%$ sodium dodecyl sulfate polyacrylamide gel electrophoresis, to the transfer onto nitrocellulose membranes (Millipore, Bedford, MA, USA), and incubated with specific primary antibodies against DCLK1 and GAPDH (Proteintech Group, Inc, PRC). Horseradish peroxidase-conjugated goat anti-rabbit IgG (Proteintech Group, Inc, PRC) and ECL detection systems (PerkinElmer, NEL100001EA, USA) were utilized to visualize the specific bands.

\section{Construction of expression vectors}

MiR-195 mimics, mimics control, miR-195 antisense oligonucleotides (miR-195 ASO), and ASO control were purchased from RiboBio. DCLK1-specific small interfering RNAs (siRNA-DCLK1) and the control small interfering RNAs (siRNA-control) were purchased from GeneChem (Shanghai, PRC). The coding sequence of DCLK1 was amplified and cloned into a pcDNA3.0 vector to generate DCLK1-expression vectors, and the empty pcDNA3.0 vector was used as control. Primers used for amplifying the DCLK1 coding sequence were 5'-GGAATTCCATGTCCTTCGGCAGAGAC-3' (forward) and 5'-CGGATCCGTCACATCCTGGTTGCGTC-3' (reverse).

\section{KARGER}




\section{Cellular Physiology Cell Physiol Biochem 2017;44:1867-1881 \begin{tabular}{l|l|l} 
and Biochemistry Published online: December 08, 2017 & $\begin{array}{l}\text { (c) } 2017 \text { The Author(s). Published by S. Karger AG, Basel } \\
\text { www.karger.com/cpb }\end{array}$ \\
\hline
\end{tabular}

\section{Cell proliferation assay}

MTT assay was performed to evaluate cell proliferation. Briefly, the PC cells were plated in 96-well plates with 5, 000 per well and were allowed to grow for 12, 24, and 48 hours. Then, the cells were assessed by a colorimetric assay using MTT solution $(10 \mathrm{mg} / \mathrm{mL})$ at $570 \mathrm{~nm}$. All the experiments were performed three times.

\section{Colony formation assay}

The transfected PC cells were harvested and seeded at a density of 200 cells per well in 12 -well plates and incubated at $37^{\circ} \mathrm{C}$ and $5 \% \mathrm{CO}_{2}$ in a humidified incubator for 2 weeks. During colony growth, the culture medium was replaced every 3 days. The colony number in each well was counted and calculated.

\section{Cell-cycle analysis by flow cytometry}

The cells were harvested by trypsinization, washed twice with cold phosphate buffered saline (PBS), and fixed in $70 \%$ ethanol overnight at $4^{\circ} \mathrm{C}$. The fixed cells were then washed with cold PBS, resuspended in staining solution ( $50 \mu \mathrm{g} / \mathrm{mL}$ of propidium iodide, $1 \mathrm{mg} / \mathrm{mL}$ of RNase A, $0.1 \%$ Triton X-100 in PBS), and incubated for 30 minutes at $4^{\circ} \mathrm{C}$. The stained cells were analyzed with a flow cytometer (Beckman Coulter Inc, Brea, CA, USA).

\section{Cell migration and invasion assay}

Cell migration was assessed by wound-healing assay. In brief, cells were seeded in 6-well plates and cultured to $100 \%$ confluence. Using a sterile pipette tip, wounds were generated and the cells were cultured for 48 hours. Then, wound closure was assessed by Scion Image software (Scion Corporation, USA).

Matrigel invasion chambers (Millipore, USA) were used to assess cell invasion ability. Briefly, $1 \times 10^{5}$ cells were seeded in the upper chamber with media containing $0.1 \%$ FBS, while the lower chamber was filled with media with $10 \%$ FBS. After incubation for 48 hours, noninvading cells were removed with cotton swabs, and cells that migrated through the membrane were fixed with $70 \%$ alcohol, stained with crystal violet and imaged, and counted under a microscope (Olympus, Japan).

\section{Tumor xenograft study in vivo}

Six-week-old male BALB/c nude mice were purchased from the Shanghai Institute for Biological Sciences (Shanghai, China). For subcutaneous xenograft, the stable miR-195-overexpression PANC-1 cells or control cells were inoculated subcutaneously in the right dorsal flank in the 7 pairs of mice with $4 \times 10^{6}$ cells per mouse. The length (L) and width (W) of the tumors were examined every 5 days with calipers and tumor volume was calculated using the formula: $\left(\mathrm{L} \times \mathrm{W}^{2}\right) / 2$. On day 25 , all mice were killed and the tumors were excised.

For detecting liver metastatic capacity, the stable miR-195-overexpression PANC-1 cells or control cells were injected into the spleen or the tail of the pancreas similarly as previously described $[15,16]$. Briefly, mice were anesthetized by continuous inhalation of isoflurane, and the abdominal wall was opened. The pancreatic tail with the spleen was gently exteriorized. $3 \times 10^{6}$ stable miR-195-overexpression PANC-1 cells or control cells in $20 \mu \mathrm{L}$ of 33\% Matrigel (Becton Dickinson, NJ, USA) were injected into the spleen or the tail of the pancreas with a 25 -gauge needle. After the pancreas was relocated into the abdominal cavity, the abdominal wall was closed with 5.0 absorbable sutures. Two months after PANC-1 cells inoculation, the experiment was terminated. The metastatic nodules in each liver were counted. The mice used in this experiment were maintained under specific pathogen-free conditions and handled in accordance with NIH Animal Care and Use Committee regulations.

\section{Statistical analysis}

Data are presented as the median \pm standard deviation. Student $t$-test was performed to compare differences in two groups. Comparisons among multiple samples were made by the Statistical Analysis System software (v.9.1.3; SAS Institute, Cary, NC, USA). Correlation between DCLK1 / miR-195 expression and clinicopathological features was estimated by the chi-square test and Pearson correlation test. KaplanMeier plots were performed with SPSS version 23.0 (SPSS Inc., Chicago, IL). All statistical tests were twosided; $P<0.05$ was considered statistically significant. 


\section{Results}

DCLK1 is upregulated in PC and high DCLK1 indicates a poor clinical outcome

In this study,
we expression of DCLK1 in 63 human PC tissues by immunohis tochemistry staining. High cytoplasmic immunoreactivity of DCLK1 was observed in tumor cells but not in the surrounding stroma. Representative staining is shown in Fig. 1A. Meanwhile, we also examined the expression levels of DCLK1 protein in differently differentiated PC tissues and adjacent healthy pancreatic tissues using western blot analysis. DCLK1 protein level was significantly increased in differently differentiated tumor tissues compared with those in the nontumor adjacent tissues. However, its level showed no difference among the differently differentiated PC tissues (Fig. 1B). The association between DCLK1 level and clinical parameters was shown in Table 1. Patients with
Table 1. Clinical characteristics of patients with $P C(n=63)$ and correlations with DCLK1 expression. * Significant difference $(\mathrm{P}<0.05)$

\begin{tabular}{|c|c|c|c|c|}
\hline Variable & Total & \multicolumn{2}{|c|}{ DCLK1 expression level } & $\mathrm{P}$-value \\
\hline & & Low & High & \\
\hline Total, $\mathrm{n}$ & 63 & & & \\
\hline Median age, years $(n \pm S D)$ & $57.35 \pm 7.51$ & & & \\
\hline Age range, years & $45-74$ & & & \\
\hline Gender, n (\%) & & & & 0.986 \\
\hline Male & $40(63)$ & 14 & 26 & \\
\hline Female & $23(37)$ & 8 & 15 & \\
\hline Histological grade, n (\%) & & & & 0.926 \\
\hline Poorly differentiated & $32(51)$ & 11 & 20 & \\
\hline Well/moderately differentiated & $31(49)$ & 11 & 21 & \\
\hline Tumour stage, n (\%) & & & & 0.224 \\
\hline $\mathrm{T} 1-\mathrm{T} 2$ & $56(89)$ & 21 & 35 & \\
\hline T3-T4 & $7(11)$ & 1 & 6 & \\
\hline TNM stage, n (\%) & & & & $0.006^{*}$ \\
\hline I + IIA & $40(63)$ & 18 & 19 & \\
\hline $\mathrm{IIB}+\mathrm{III}+\mathrm{IV}$ & $23(37)$ & 4 & 22 & \\
\hline Lymph node metastasis, n (\%) & & & & $0.002 *$ \\
\hline Yes & $21(33)$ & 2 & 20 & \\
\hline No & $42(67)$ & 20 & 21 & \\
\hline
\end{tabular}

Table 2. Clinical characteristics of patients with PC $(n=42)$ and correlations with miR-195 expression. * Significant difference $(\mathrm{P}<0.05)$

\begin{tabular}{|c|c|c|c|c|}
\hline Variable & Total & \multicolumn{2}{|c|}{ miR-195 expression level } & $\mathrm{P}$-value \\
\hline & & High & Low & \\
\hline Total, $\mathrm{n}$ & 42 & & & \\
\hline Median age, years $(n \pm S D)$ & $57.26 \pm 7.76$ & & & \\
\hline Age range, years & $45-72$ & & & \\
\hline Gender, $\mathrm{n}(\%)$ & & & & 0.372 \\
\hline Male & 26 & 10 & 14 & \\
\hline Female & 16 & 10 & 8 & \\
\hline Histological grade, $\mathrm{n}(\%)$ & & & & 0.516 \\
\hline Poorly differentiated & 20 & 8 & 11 & \\
\hline Well/moderately differentiated & 22 & 12 & 11 & \\
\hline Tumour stage, n (\%) & & & & 0.188 \\
\hline $\mathrm{T} 1-\mathrm{T} 2$ & 39 & 19 & 18 & \\
\hline T3-T4 & 3 & 1 & 4 & \\
\hline TNM stage, $\mathrm{n}(\%)$ & & & & $0.012^{*}$ \\
\hline I + IIA & 30 & 15 & 8 & \\
\hline IIB + III + IV & 12 & 5 & 14 & \\
\hline Lymph node metastasis, n (\%) & & & & $0.021 *$ \\
\hline Yes & 12 & 4 & 12 & \\
\hline No & 30 & 16 & 10 & \\
\hline
\end{tabular}

higher DCLK1 level had a higher TNM stage and a higher rate of lymph node metastasis compared with those with lower DCLK1 level. In addition, the high DCLK1 expression level was correlated with poorer prognosis (Fig. 1C). These results indicate that DCLK1 may play a tumor-promoting effect in PC.

MiR-195 is downregulated in PC and negatively correlated with DCLK1 level, and low miR195 indicates a poor clinical outcome

By qRT-PCR assay, we found that miR-195 level was significantly reduced in differently differentiated tumor tissues compared with that in the non-tumor adjacent tissues, and its level in the poorly differentiated PC tissues was lower than that in the well and moderately differentiated tissues (Fig. 2A). Moreover, we found that miR-195 exhibited a lower level in all the PC cell lines except AsPC-1 compared with the HPDE6-C7 normal PC cells (Fig. 2B). MiR-195 also showed a negative correlation with DCLK1 level (Fig. 2C) and lower miR195 level is associated with higher TNM stage, higher rate of lymph node metastasis, and poorer prognosis (Table 2 and Fig. 2D). These data suggest that decreased miR-195 may be associated with PC. 


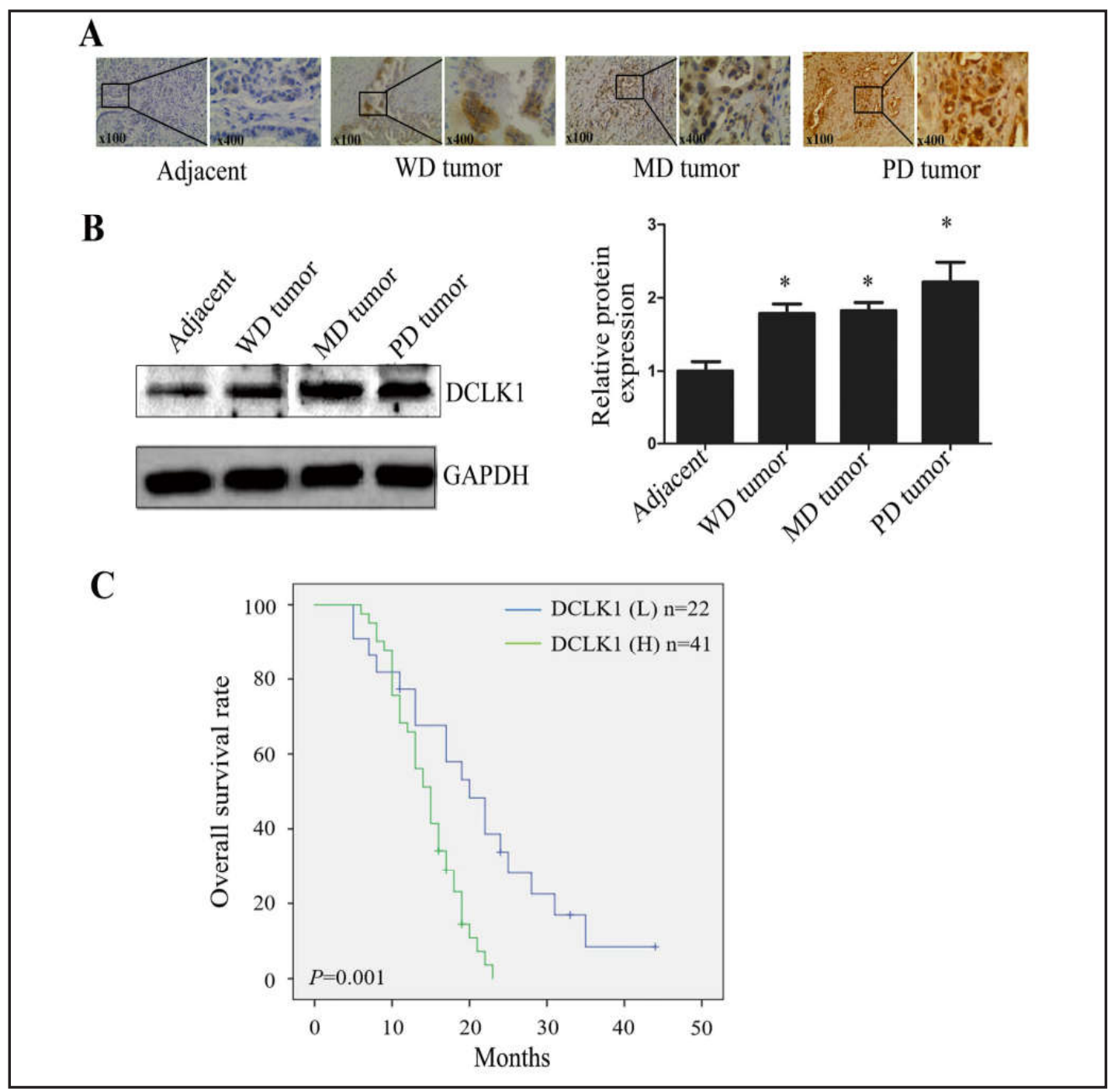

Fig. 1. The expression of DCLK1 in PC tissues and adjacent normal tissues. Immunohistochemical staining (A) and western blot analysis (B) were performed to detect the DCLK1 protein level in well-differentiated human PC tissues (WD tumors, $\mathrm{n}=11$ ), moderately differentiated PC tissues (MD tumors, $\mathrm{n}=14$ ), poorly differentiated PC tissues (PD tumors, $n=6$ ) and adjacent healthy pancreatic tissues ( $n=9)($ Left: $\times 100$, Right: $\times 400$ ). (C) Kaplan-Meier analysis was performed to analyze the overall survival of PC patients with high and low expression levels of DCLK1 $(n=63) .{ }^{*} \mathrm{P}<0.05$.

\section{MiR-195 directly inhibits DCLK1 expression}

To expound the molecular mechanism underlying DCLK1 exerting its effects on PC cells, we predicted potential miRNA of DCLK1 using the TargetScan, miRanda, and PicTar online tools, and identified two conserved binding sites of miR-195 in the $3^{\prime}$ untranslated region (UTR) of the DCLK1 mRNA. We speculated that DCLK1 was a potential target for miR-195. To confirm this, we first conducted a luciferase reporter assay. A fragment of wild-type or mutant 3' UTR of DCLK1 was cloned into the psiCheck-2 reporter vector (Fig. 3A). Our results showed that $m i R-195$ mimics significantly decreased the firefly luciferase activity of the vector with the wild-type $3^{\prime}$ UTR of DCLK1, but had no significant effect on the vector with the mutated 3' UTR of DCLK1 (Fig. 3B). Further, we detected the basic levels of DCLK1 mRNA and protein in the four PC cell lines including PANC-1, BxPC-3, SW1990, and AsPC-1. The results showed that DCLK1 mRNA and protein levels were significantly lower in AsPC-1 


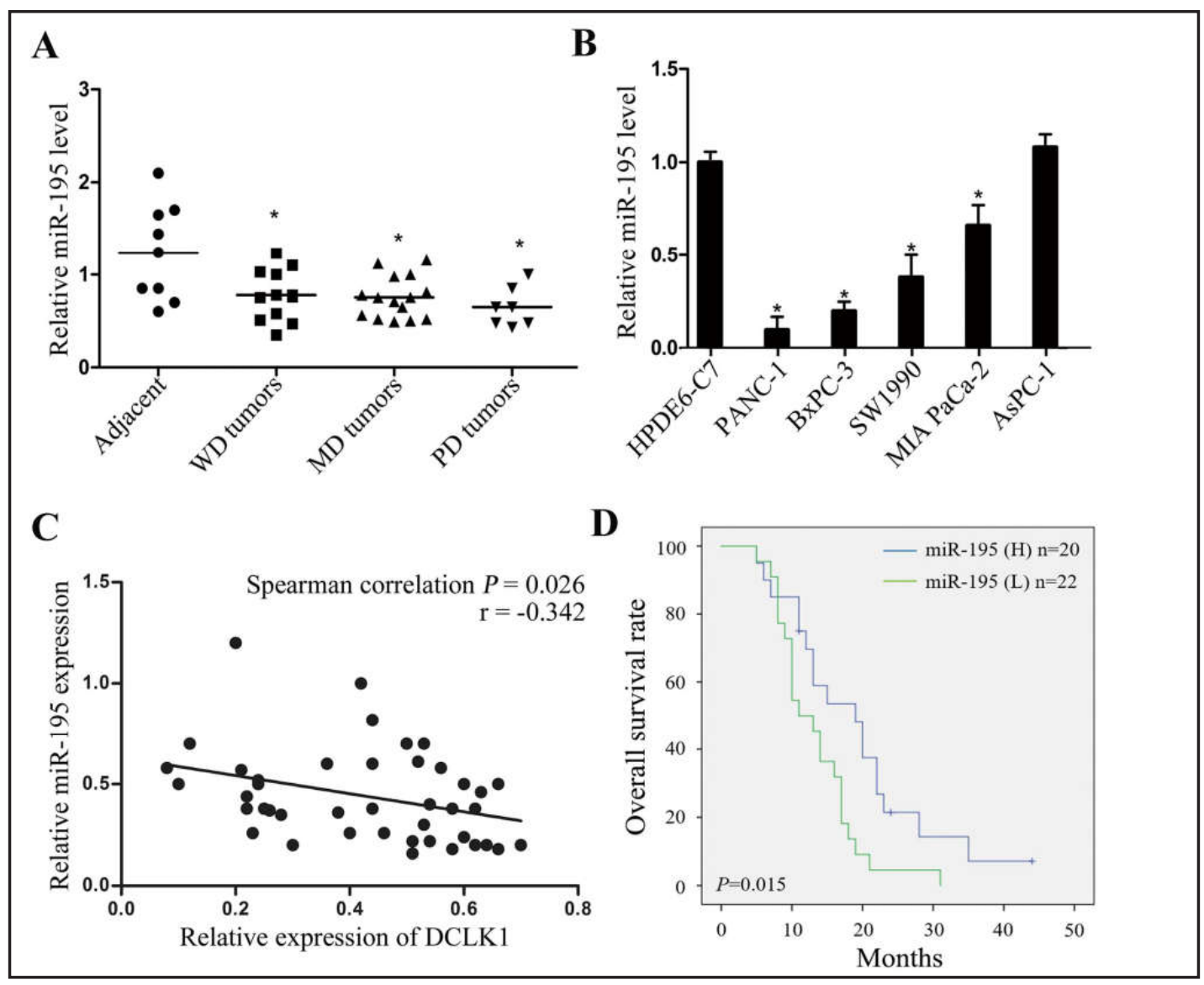

Fig. 2. MiR-195 expression in PC tissues and cell lines and its correlation with DCLK1 and prognosis. (A) miR-195 level was evaluated by qPCR in well-differentiated human PC tissues (WD tumors, $\mathrm{n}=11$ ), moderately differentiated PC tissues (MD tumors, $n=14$ ), poorly differentiated PC tissues (PD tumors, $\mathrm{n}=6$ ) and adjacent normal tissues $(\mathrm{n}=9$ ). (B) miR-195 level in PC cell lines (BxPC-3, PANC-1, SW1990, MIAPaCa-2, and AsPC-1) and normal human pancreatic duct epithelial cells (HPDE6-C7) was detected by qPCR. (C) The correlation between miR-195 and DCLK1 in differently differentiated PC tissues and adjacent normal tissues was analyzed. (D) Kaplan-Meier analysis was performed to analyze the overall survival of PC patients with high and low expression levels of miR-195 $(n=63) .{ }^{*}<<0.05$.

cell line than those in the other PC cell lines. Furthermore, we found that DCLK1 mRNA and protein levels in the miR-195 mimics treated PANC-1, BxPC-3, and SW1990 cells were lower than the control cells. Because the relative level of $m i R-195$ in AsPC-1 cells was high (Fig. 2B), we transfected miR-195 ASO into the AsPC-1 cells to suppress endogenous miR-195. We observed increased DCLK1 mRNA and protein levels in the miR-195 ASO treated AsPC-1 cells (Fig. 3C and 3D). These results demonstrated that miR-195 can directly inhibit DCLK1 expression by binding to its $3^{\prime}$ UTR in PC cells.

MiR-195 inhibits the proliferation, migration and invasion of PC cells in vitro and in vivo

To identify the effects of miR-195 on PC cells, miR-195 mimics or miR-195 ASO was transfected into PC cells to alter miR-195 level, and qRT-PCR confirmed miR-195's elevation in PANC-1 cells and its reduction in AsPC-1 cells (Fig. 4A). As shown in Fig. 4B and 4C, overexpression of $m i R-195$ was inhibited, whereas downregulation of $m i R-195$ promoted the proliferation of AsPC- 1 cells by MTT assay. Furthermore, the colony number of the in vitro cultured PC cells was negatively correlated with the expression level of miR-195 (Fig. 4D). To reveal the mechanisms of miR-195 on suppressing the tumor, we analyzed the PC cell cycle 


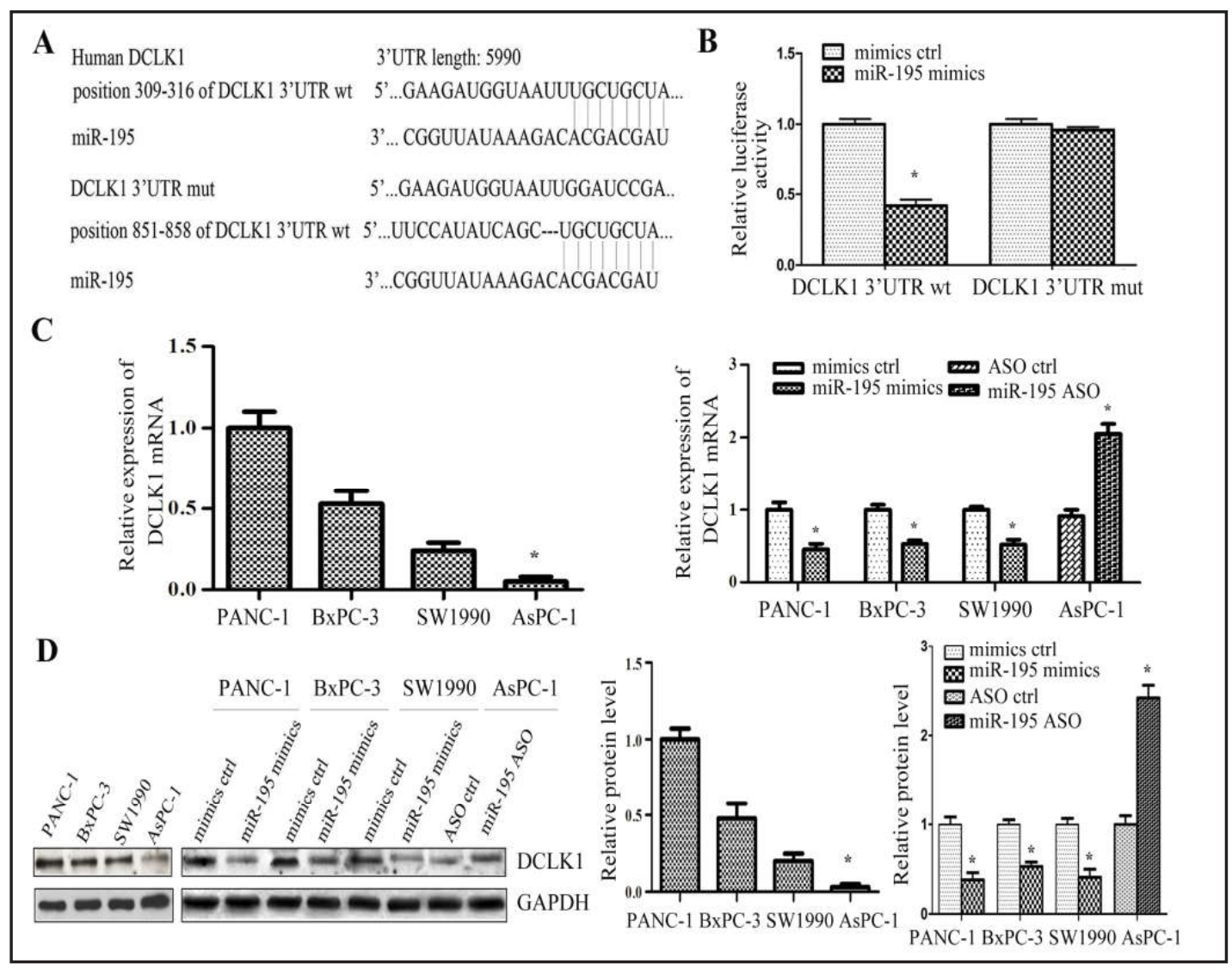

Fig. 3. MiR-195 inhibits DCLK1 expression in PC cells. (A) Predicted binding sequences of miR-195 in DCLK1 3' UTR is shown. The fragment of wild-type (wt) or mutant (mut) 3' UTR of DCLK1 was cloned into the luciferase reporter vector. (B) PANC-1 cells were co-transfected with miR-195 mimics and luciferase reporters containing wild type (wt) or mutated (mut) DCLK1 mRNA 3' UTR. Luciferase activities measured at 48 hours posttransfection. The DCLK1 mRNA (C) and protein (D) level in 4 PC cell lines (PANC-1, BxPC-3, SW1990, and AsPC-1), miR-195 mimics transfected PANC-1, BxPC-3, SW1990, and miR-195 ASO transfected AsPC-1 cells was measured by qRT-PCR and Western blot, respectively. ${ }^{*} \mathrm{P}<0.05$.

using flow cytometry. As shown in Fig. 4E, overexpression of miR-195 significantly increased the proportion of G1 phases and reduced the proportion of G2 and S phases in PANC-1 cells, and the miR-195 ASO resulted in an opposite phenomenon in the AsPC-1 cells. In addition, the mean volumes of xenograft tumors derived from the PANC- 1 cells stably overexpressing miR-195 were smaller than those originated from the control cells $\left(P=4.68 \times 10^{-3}\right)$ (Fig. 4F and 4G). Collectively, these data suggest that miR-195 inhibits PC cell growth in vitro and in vivo.

The effects of miR-195 on PC cell invasion and migration were investigated by transwell and wound-healing assays. We found that overexpression of miR-195 significantly inhibited cell invasion and migration ability in PANC-1 cells. In contrast, after endogenous miR-195 was inhibited by miR-195 ASO, there was an obvious increase in invasion and migration ability of AsPC-1 cells (Fig. 4H and 4I). More importantly, animal experiments showed that overexpression of miR-195 dramatically alleviated PANC-1 cells metastasis in vivo (Fig. 4J). These results revealed that miR-195 suppresses PC cell migration, invasion, and metastasis. 


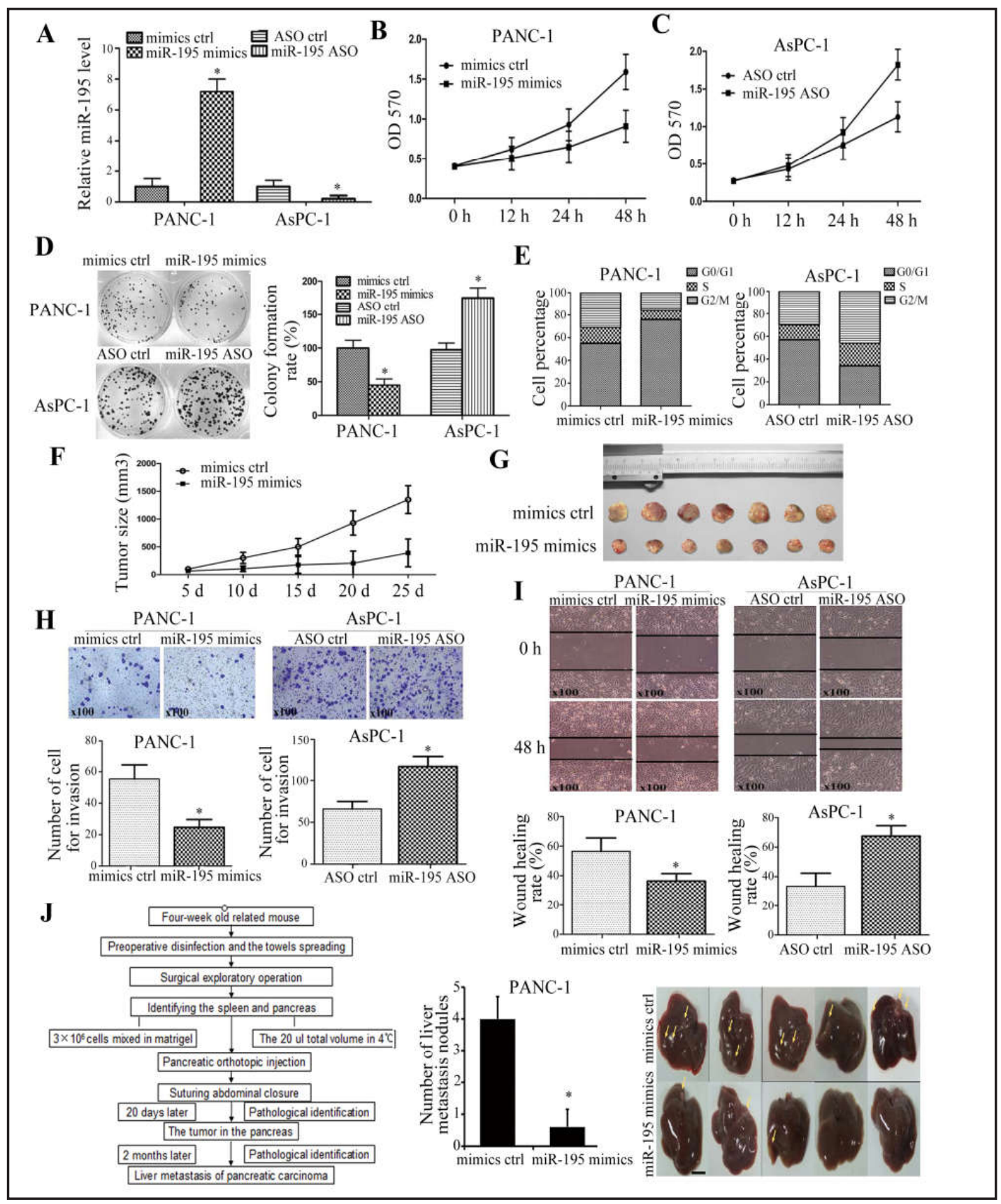

Fig. 4. MiR-195 inhibits the proliferation, migration and invasion of PC cells in vitro and in vivo. (A) miR-195 mimics or ASO was transfected into PANC-1 or AsPC-1 cells, respectively, and miR-195 level measured using qRT-PCR. (B, C) The cell viability of PANC-1 cells transfected with miR-195 mimics (B) and AsPC-1 cells transfected with miR-195 ASO (C) was detected using MTT assay. (D) The colony formation of the transfected PANC-1 and AsPC-1 cells was detected. (E) The effect of miR-195 on PC cell cycle was determined using flow cytometry. (F) The growth of xenograft tumors derived by miR-195-treated PC cells was determined in vivo. (G) The representative image of the xenograft tumors is shown ( $n=7$ per group). The effects of miR-195 on cell invasion and migration were determined using transwell assays $(\mathrm{H})$ and wound-healing assays (I), respectively $(\times 100)$. (J) The schematic representation of the experimental and representative liver metastatic tumors derived by miR-195- treated PC cells in mice. The histogram shows the average number of tumors in the liver ( $\mathrm{n}=$ five per group; Column: mean; bars: \pm standard deviation. Scale bars, 5 $\mathrm{mm})$. ${ }^{*} \mathrm{P}<0.05$.

\section{KARGER}




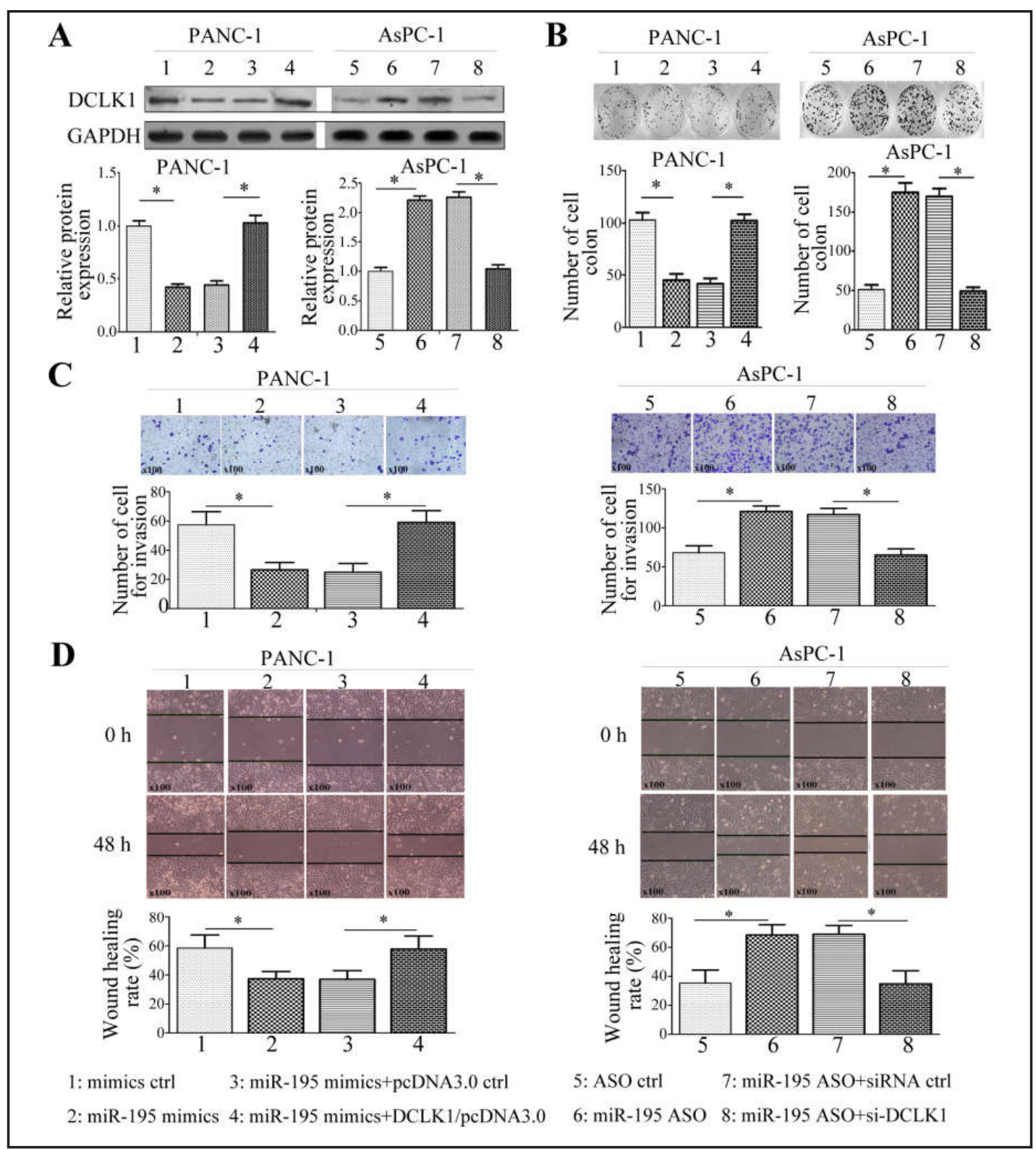

Fig. 5. MiR-195 performs its roles in PC cells by downregulating DCLK1 expression. (A) The DCLK1 protein level in the AsPC-1 cells cotransfected with miR-195 ASO and si-DCLK1, and in the PANC-1 cells cotransfected with miR-195 mimics and DCLK1 overexpression vector was measured by western blot analysis. (B) The colony formation of the transfected PANC-1 and AsPC-1 cells was detected. The effects of miR-195 on cell invasion and migration were determined using transwell assays (C) and wound-healing assays (D), respectively $(\times 100) . * \mathrm{P}<0.05$.

MiR-195 performs its tumor suppressor role in PC cells by downregulating DCLK1 expression

DCLK1 protein is involved in the growth, invasion, and metastasis of the PC cells [17, 18]. In this research, short hairpin RNA targeting DCLK1 mRNA (si-DCLK1) was exploited to specifically inhibit the expression of DCLK1 in miR-195 ASO treated AsPC-1 cells. The $D C L K 1 /$ pcDNA3.0 vector was used to overexpress DCLK1 in miR-195 mimics treated PANC-1 cells. As shown in Fig. 5A, si-DCLK1 reduced the high $D C L K 1$ protein level caused by miR-195 
A

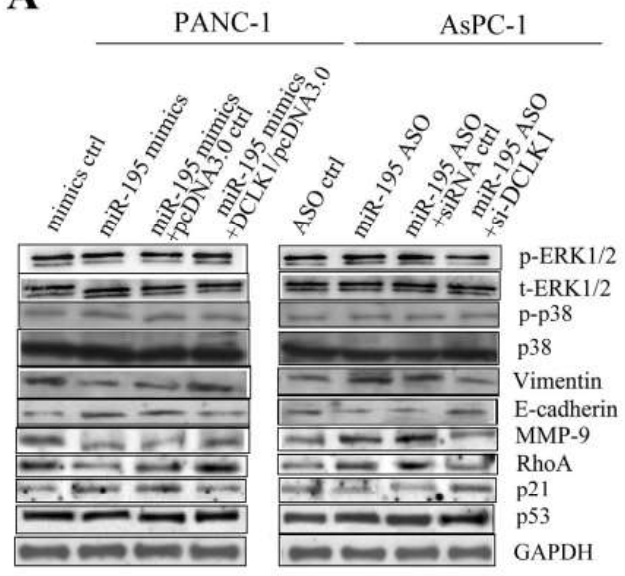

B

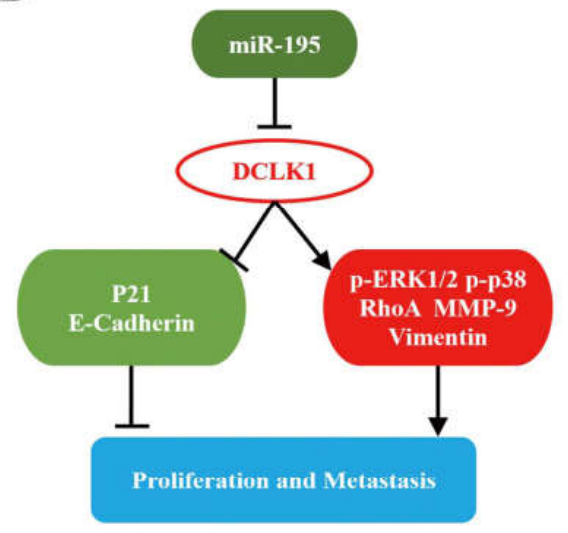

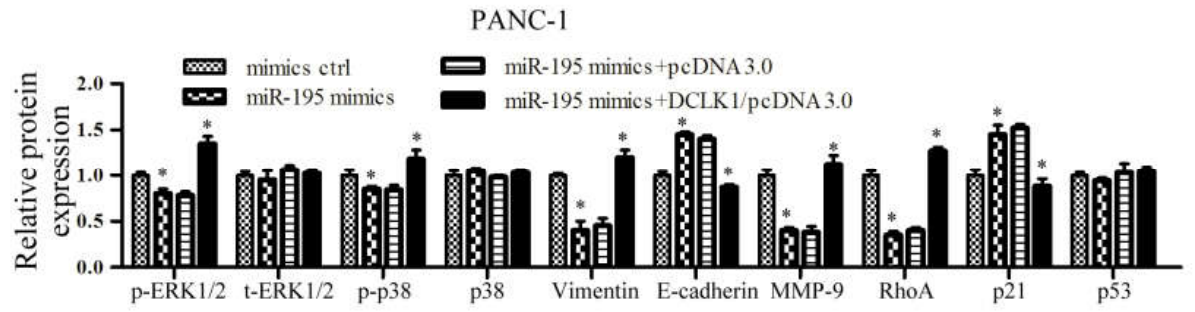

AsPC-1

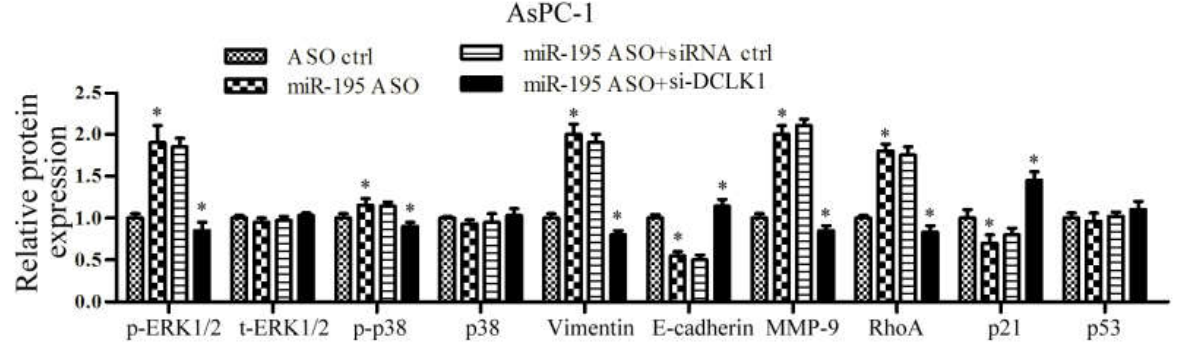

Fig. 6. MiR-195 regulates the expression of p-ERK1/2, p-p38, p21, vimentin, E-cadherin, RhoA, and MMP9 by targeting DCLK1. (A) Effects of miR-195 on p-ERK1/2, t-ERK1/2, p-p38, p38, vimentin, E-cadherin, MMP-9, RhoA, p53, and p21 were analyzed by western blot analysis in the transfected PC cells. (B) miR-195 inhibits DCLK1 via upregulation of p21 and E-cadherin, and downregulation of p-ERK1/2, p-p38, vimentin, RhoA, and MMP-9, which results in inhibition of proliferation and metastasis in PC cells. * $\mathrm{P}<0.05$.

ASO in AsPC-1 cells. Further, DCLK1 overexpression vector prevented the reduction of $D C L K 1$ protein level caused by $m i R-195$ mimics. These results support the point that miR-195 plays the same role as si-DCLK1 in PC cells and serves as a negative regulator of $D C L K 1$ expression. Inhibition or overexpression of DCLK1 also reversed the changes of colony formation, migration, and invasion of PC cells induced by miR-195 ASO or mimics, respectively (Fig. $5 B, 5 C$, and 5D). These results suggest that miR-195 inhibits PC cell malignancy through targeting DCLK1.

MiR-195 regulates $p$-ERK1/2, p-p38, p21, vimentin, E-cadherin, RhoA, and MMP-9 expression in PC cells by targeting DCLK1

To investigate the mechanisms of miR-195 in regulation of PC cells, we analyzed the effects of miR-195 on the proliferation-, invasion- and migration-related genes, such as p-ERK1/2, p-p38, p21, vimentin, E-cadherin, RhoA, and MMP-9. As shown in Fig. 6A, miR-195 mimics transfected PANC-1 cells had low levels of $p$-ERK1/2, $p$ - $p 38$, vimentin, RhoA, and MMP- 
9, and increased levels of $p 21$ and E-cadherin. Meanwhile, cotransfection of miR-195 mimics and DCLK1/pcDNA3.0 vector into PANC-1 cells resulted in increased levels of $p$-ERK1/2, $p$-p38, vimentin, RhoA, and MMP-9, and decreased levels of $p 21$ and E-cadherin. Furthermore, downregulation of $m i R-195$ and knockdown of DCLK1 resulted in opposite changes of these genes in AsPC-1 cells. However, miR-195 had no significant effect on expression of $p 53, p 38$, and $t-E R K 1 / 2$.

\section{Discussion}

DCLK1 (formerly known as DCAMKL1) is a putative intestinal and pancreatic stem cell marker and is upregulated in the stroma and epithelium of PC. It has also recently been described as a marker of the relatively undefined tuft/brush cell in the intestine and pancreas $[19,20]$. Some studies have shown that DCLK1 expression was detected at a high level in gastric cancer [21], esophageal cancer [22], and colorectal cancer [23]. We previously found a mutation of DCLK1 in PC tissues, especially in liver metastatic lesions, and a high DCLK1 expression level in PC tissues. In vitro studies indicated that DCLK1 might have promoting functional roles in PC cells [6]. This finding was confirmed by other studies. Ito et al. found that DCLK1 was overexpressed in the metastatic tumors in PC patients, and overexpression of DCLK1 promoted the migration of PC cells. Knockdown of DCLK1 profoundly suppressed in vivo liver metastasis of PC cells [17]. In this study, we discovered that DCLK1 is not only upregulated in PC tissues but also positively correlated with TNM stage, lymph node metastasis, and poorer prognosis of patients with PC. This means that the high expression of DCLK1 might be closely related to the progression and metastasis of PC.

The dysregulation of miRNA is implicated in the development and progression of practically all tumor types [24]. However, the function and mechanism of these deregulated miRNAs in various cancers including PC remain undefined. To date, various miRNAs (including $m i R-21$, $m i R-34 a, m i R-155$, and $m i R-29 a$ ) has been shown to be associated with tumor progression and overall survival in patients with PC [10, 25]. Most miRNAs are known to regulate target mRNAs by binding to the $3^{\prime}$ UTR of target genes in a posttranscriptional manner. Thus, establishing the interrelationship of miRNA and its target genes may help improve understanding of the molecular mechanism underlying cancer progression and provide potential therapeutic targets for the clinical treatment of cancers. Our study showed that the levels of DCLK1 mRNA and protein are both negatively regulated by miR-195 in PC cells. The direct targeting was further supported through luciferase reporter assay. These results indicate that miR-195 might influence the function of PC cells by targeting DCLK1.

Recently, compelling evidence has demonstrated that miR-195 is associated with the biological features of cancers, such as oncogenesis, development, and metastasis. miR-195 is frequently silenced or downregulated in many types of cancers, including prostate cancer [11], non-small cell lung cancer [12], hepatocellular cancer [14], gastric cancer [26], glioma [27], osteosarcoma [28], and colorectal cancer [29]. In these cancers, miR-195 usually has tumor-suppressive effects. In this study, we found that the expression level of miR-195 was low in PANC-1, BxPC-3, SW1990, and MIAPaCa-2 cells; however, it was high in AsPC-1 cells. This result suggested that the different expression level of miR-195 is related to the heterogeneity and complexity of PC cells.

It has been previously demonstrated that antigrowth signals prevent cell proliferation through two different mechanisms. Cells may enter the quiescent state (G0) and not participate in the active proliferative cycle; or cells may be induced to enter postmitotic states and lose the potential to differentiate [30]. In the current study, overexpression of miR-195 in PANC-1 cells may result in the activation of antigrowth signals, inducing cell cycle arrest at the G0/G1 phase and disturbance to the G1 to S phase transition. Furthermore, we found that overexpression of miR-195 suppressed the invasion and migration ability of PANC-1 cells, and downregulation of miR-195 resulted in the opposite phenomenon in AsPC-1 cells using transwell and wound-healing assays. In addition, we demonstrated that tumor growth 


\section{Cellular Physiology Cell Physiol Biochem 2017;44:1867-1881 \begin{tabular}{l|l|l} 
DOI: 10.1159/000485876 & O 2017 The Author(s). Published by S. Karger AG, Basel \\
www.karger.com/cpb
\end{tabular} \\ Zhou et al.: MiR-195 Targets DCLK1 in PC}

and metastasis in the nude mice were well inhibited by overexpressed miR-195 in PANC-1 cells. Lower expression of miR-195 in human PC tissues is also associated with a higher TNM stage, higher rate of lymph node metastasis, and poorer prognosis of PC patients. On the basis of these results, we considered that miR-195 may serve as a negative regulator in the tumorigenesis and development of PC.

However, some studies found that miR-195 increased in the circulation of breast cancer and chronic lymphocytic leukemia patients compared with those corresponding control subjects $[31,32]$. In this research, we also observed that miR-195 expression significantly decreased in differently differentiated PC tissues and cell lines. But it is not downregulated in AsPC-1 cells, suggesting that the role of miR-195 in different PC cell lines is distinguishing. As a result, these data suggest that the roles of miR-195 might vary in different types of cancers.

In our analysis of clinical human PC tissues, the DCLK1 mRNA level was negatively correlated with miR-195 level. Our functional studies also revealed that the DCLK1 was positively correlated with cell proliferation, migration, and invasion of PC. Therefore, these results confirmed DCLK1 as a functional target of miR-195 in PC cells and validated the function of miR-195-DCLK1 interaction in the progression of PC.

To investigate the molecular mechanisms of miR-195 in inhibiting DCLK1 in PC cells, we analyzed the known proliferation-, invasion- and migration-related genes. It has been recently reported that activated $p$-ERK and $p$ - $p 38 M A P K$ signaling pathways and E-cadherin take part in regulating proliferation, migration, and invasion of PANC-1 cells [33]. In addition, downregulation of vimentin suppresses the proliferation and invasion of PC cells in vitro and in vivo [34]. Oncogenic K-Ras enhances the malignant phenotype and the mitogen-activated protein kinase $p 38$ was identified as a target to inhibit oncogenic $K$-Ras-induced pancreatic cancer cell migration [35]. Moreover, the expression of $M M P-9$, which was correlated with vascular invasion, lymph node invasion, liver metastases, and TNM stage, was increased in PC [36]. Thus, our discovery demonstrated that abnormal alteration of miR-195-DCLK1 interaction might occur through regulating $p$-ERK1/2, p-p38, p21, vimentin, E-cadherin, RhoA, and $M M P-9$, which contribute to the progression and metastasis of PC cells (Fig. 6B).

\section{Conclusion}

In summary, our results revealed that miR-195 is significantly downregulated in PC and plays tumor suppressor roles in inhibiting the proliferation, migration, and invasion of PC cells by negatively regulating DCLK1. These data suggest that the miR-195 targeting DCLK1 pathway in tumor growth and metastasis may provide a novel possibility to diagnose and treat $\mathrm{PC}$ in the future.

\section{Acknowledgements}

This work was supported by the National Natural Science Foundation of China (81302077). We thank all the patients and clinical investigators who were involved in this study.

\section{Disclosure Statement}

The authors declare no conflicts of interest. 


\section{Cellular Physiology Cell Physiol Biochem 2017;44:1867-1881 \begin{tabular}{l|l|l} 
and Biochemistry Published online: December 08, 2017 & $\begin{array}{l}\text { (c) } 2017 \text { The Author(s). Published by S. Karger AG, Basel } \\
\text { www.karger.com/cpb }\end{array}$ \\
\hline
\end{tabular}

\section{References}

1 Siegel RL, Miller KD, Jemal A: Cancer statistics, 2016 CA Cancer J Clin 2016;66:7-30.

2 Maitra A, Hruban RH: Pancreatic cancer. Annu Rev Pathol 2008;3:157-188.

-3 Bussom S, Saif MW: Methods and rationale for the early detection of pancreatic cancer. Highlights from the "2010 asco gastrointestinal cancers symposium". Orlando, fl, USA. January 22-24, 2010. JOP 2010;11:128130.

4 Shahrokni A, Saif MW: Metastatic pancreatic cancer: The dilemma of quality vs. Quantity of life. JOP 2013;14:391-394.

-5 Garridolaguna I, Hidalgo M: Pancreatic cancer: From state-of-the-art treatments to promising novel therapies. Nat Rev Clin Oncol 2015;12:319-334.

-6 Zhou B, Irwanto A, Guo YM, Bei JX, Wu Q Chen G, Zhang TP, Lei JJ, Feng QS, Chen LZ: Exome sequencing and digital PCR analyses reveal novel mutated genes related to the metastasis of pancreatic ductal adenocarcinoma. Cancer Biol Ther 2012;13:871-879.

7 Bailey JM, Alsina J, Rasheed ZA, Mcallister FM, Fu YY, Plentz R, Zhang H, Pasricha PJ, Bardeesy N, Matsui W: DCLK1 marks a morphologically distinct subpopulation of cells with stem cell properties in preinvasive pancreatic cancer. Gastroenterology 2014;146:245-256.

-8 Sureban SM, May R, Lightfoot SA, Hoskins AB, Lerner M, Brackett DJ, Postier RG, Ramanujam R, Mohammed A, Rao CV: DCAMKL-1 regulates epithelial-mesenchymal transition in human pancreatic cells through a mir-200a-dependent mechanism. Cancer Res 2011;71:2328-2338.

-9 Sureban SM, May R, Ramalingam S, Subramaniam D, Natarajan G, Anant S, Houchen CW: Selective blockade of DCAMKL-1 results in tumor growth arrest by a let-7a microrna-dependent mechanism. Gastroenterology 2009;137:649-659.

10 Sun XJ, Liu BY, Yan S, Jiang TH, Cheng HQ, Jiang HS, Cao Y, Mao AW: MicroRNA-29a promotes pancreatic cancer growth by inhibiting tristetraprolin. Cell Physiol Biochem 2015;37:707-718.

-11 Cai C, Chen QB, Han ZD, Zhang YQ He HC, Chen JH, Chen YR, Yang SB, Wu YD, Zeng YR: Mir-195 inhibits tumor progression by targeting RPS6KB1 in human prostate cancer. Clin Cancer Res 2015;21:4922-4934.

-12 Liu B, Qu J, Xu F, Guo Y, Wang Y, Yu H, Qian B: MiR-195 suppresses non-small cell lung cancer by targeting CHEK1. Oncotarget 2015;6:9445-9456.

13 Jain M, Zhang L, Boufraqech M, Liu-Chittenden Y, Bussey K, Demeure MJ, Wu X, Su L, Pacak K, Stratakis CA, Kebebew E: ZNF367 inhibits cancer progression and is targeted by miR-195. PLoS One 2014;9:e101423.

-14 Wang R, Zhao N, Li S, Fang JH, Chen MX, Yang J, Jia WH, Yuan Y, Zhuang SM: MicroRNA-195 suppresses angiogenesis and metastasis of hepatocellular carcinoma by inhibiting the expression of VEGF, VAV2, and CDC42. Hepatology 2013;58:642-653.

15 Zhou B, Zhan H, Tin L, Liu S, Xu J, Dong Y, Li X, Wu L, Guo W: TUFT1 regulates metastasis of pancreatic cancer through HIF1-Snail pathway induced epithelial-mesenchymal transition. Cancer Lett 2016;382:1120.

16 Zhou B, Wu Q, Chen G, Zhang TP, Zhao YP: NOP14 promotes proliferation and metastasis of pancreatic cancer cells. Cancer Lett 2012;322:195-203.

17 Ito H, Tanaka S, Akiyama Y, Shimada S, Adikrisna R, Matsumura S, Aihara A, Mitsunori Y, Ban D, Ochiai T, Kudo A, Arii S, Yamaoka S: Dominant Expression of DCLK1 in Human Pancreatic Cancer Stem Cells Accelerates Tumor Invasion and Metastasis. PLoS One 2016;11:e0146564.

-18 Sureban SM, May R, Qu D, Weygant N, Chandrakesan P, Ali N, Lightfoot SA, Pantazis P, Rao CV, Postier RG, Houchen CW: DCLK1 regulates pluripotency and angiogenic factors via microRNA-dependent mechanisms in pancreatic cancer. PLoS One 2013;8:e73940.

19 Gerbe F, van Es JH, Makrini L, Brulin B, Mellitzer G, Robine S, Romagnolo B, Shroyer NF, Bourgaux JF, Pignodel C, Clevers H, Jay P: Distinct ATOH1 and Neurog3 requirements define tuft cells as a new secretory cell type in the intestinal epithelium. J Cell Biol 2011;192:767-780.

-20 Bjerknes M, Khandanpour C, Moroy T, Fujiyama T, Hoshino M, Klisch TJ, Ding Q Gan L, Wang J, Martin MG, Cheng H: Origin of the brush cell lineage in the mouse intestinal epithelium. Dev Biol 2012;362:194-218.

21 Nam KT, O’Neal R, Lee YS, Lee YC, Coffey RJ, Goldenring JR: Gastric tumor development in Smad3deficient mice initiates from forestomach/glandular transition zone along the lesser curvature. Lab Invest 2012;92:883-895. 


\section{Cellular Physiology Cell Physiol Biochem 2017;44:1867-1881 \begin{tabular}{l|l} 
DOI: 10.1159/000485876 & $\begin{array}{l}\text { O 2017 The Author(s). Published by S. Karger AG, Basel } \\
\text { www.karger.com/cpb }\end{array}$
\end{tabular} \\ Zhou et al.: MiR-195 Targets DCLK1 in PC}

22 Vega KJ, May R, Sureban SM, Lightfoot SA, Qu D, Reed A, Weygant N, Ramanujam R, Souza R, Madhoun M, Whorton J, Anant S, Meltzer SJ: Identification of the putative intestinal stem cell marker doublecortin and CaM kinase-like-1 in Barrett's esophagus and esophageal adenocarcinoma. J Gastroenterol Hepatol 2012;27:773-780.

23 Gagliardi G, Goswami M, Passera R, Bellows CF: DCLK1 immunoreactivity in colorectal neoplasia. Clin Exp Gastroenterol 2012;5:35-42.

24 Hammond SM: MicroRNAs as oncogenes. Curr Opin Genet Dev 2006;16:4-9.

25 Song W, Li Q Wang L, Wang L: Modulation of Fox01 expression by miR-21 to promote growth of pancreatic ductal adenocarcinoma. Cell Physiol Biochem 2015;35:184-190.

-26 Deng H, Guo Y, Song H, Xiao B, Sun W, Liu Z, Yu X, Xia T, Cui L, Guo J: MicroRNA-195 and microRNA-378 mediate tumor growth suppression by epigenetical regulation in gastric cancer. Gene 2013;518:351-359.

-27 Wang H, Lu Y, Luo L, Li WS, Liang CF, He HY, Ba Y: MicroRNA-195 inhibits the proliferation of human glioma cells by directly targeting cyclin D1 and cyclin E1. PLoS One 2013;8:e54932.

28 Mao JH, Zhou RP, Peng AF, Liu ZL, Huang SH, Long XH, Shu Y: microRNA-195 suppresses osteosarcoma cell invasion and migration in vitro by targeting FASN. Oncol Lett 2012;4:1125-1129.

29 Yang B, Tan Z, Song Y: Study on the molecular regulatory mechanism of MicroRNA-195 in the invasion and metastasis of colorectal carcinoma. Int J Clin Exp Med 2015;8:3793-3800.

30 Hanahan D, Weinberg RA: The hallmarks of cancer. Cell 2000;100:57-70.

-31 Heneghan HM, Miller N, Lowery AJ, Sweeney KJ, Newell J, Kerin MJ: Circulating micrornas as novel minimally invasive biomarkers for breast cancer. Ann Surg 2010;251:499-505.

-32 Zanette DL, Rivadavia F, Molfetta GA, Barbuzano FG, Protosiqueira R, Silvajr WA, Falcão RP, Zago MA: Mirna expression profiles in chronic lymphocytic and acute lymphocytic leukemia. Braz J Med Biol Res 2007;40:1435-1440.

-33 Cao L, Chen X, Xiao X, Ma Q, Li W: Resveratrol inhibits hyperglycemia-driven ROS-induced invasion and migration of pancreatic cancer cells via suppression of the ERK and p38 MAPK signaling pathways. Int J Oncol 2016;49:735-743.

34 Zhu S, He C, Deng S, Li X, Cui S, Zeng Z, Liu M, Zhao S, Chen J, Jin Y: Mir-548an, transcriptionally downregulated by HIF-1 $\alpha$ /HDAC1, suppresses tumorigenesis of pancreatic cancer by targeting vimentin expression. Mol Cancer Ther 2016;15:2209-2219.

-35 Dreissigacker U, Mueller MS, Unger M, Siegert P, Genze F, Gierschik P, Giehl K: Oncogenic K-Ras downregulates Rac1 and RhoA activity and enhances migration and invasion of pancreatic carcinoma cells through activation of p38. Cell Signal 2006;18:1156-1168.

36 Xu Y, Li Z, Jiang P, Wu G, Chen K, Zhang X, Li X: The co-expression of MMP-9 and Tenascin-C is significantly associated with the progression and prognosis of pancreatic cancer. Diagn Pathol 2015;10:211. 